\title{
Preface: impacts of CKD clinical practice guidebook on Asian CKD patients
}

Global statistics clearly and definitely show the importance of chronic kidney disease (CKD) in terms of the increase not only of dialysis patients but also of cardio-vascular diseases (CVD), including myocardial infarction and cerebral infarction.

This guidebook on CKD prevention, diagnosis, and treatment is primarily intended to help general practitioners identify patients with risk factors of CKD, and early CKD patients. The contents of this book comprise the definition, prevention, diagnosis, follow-up, and treatments of CKD.
The English version of the CKD guidebook is translated from the original Japanese version in an attempt to become an invaluable tool in Asian countries, including Japan, with similar culture and ethos. Therefore, we are sure this guidebook will be helpful for prevention, diagnosis, and treatment of Asian CKD patients.

Yasuhiko Iino, $\mathrm{MD}, \mathrm{PhD}$

Leader of the CKD Clinical Practice Guidebook Production Group 